\title{
Quantum state transmission over partially corrupted quantum information network
}

\author{
Masahito Hayashi $\odot^{1,2,3,4, *}$ and Seunghoan Song $\oplus^{4, \dagger}$ \\ ${ }^{1}$ Shenzhen Institute for Quantum Science and Engineering, Southern University of Science and Technology, Shenzhen 518055, China \\ ${ }^{2}$ Guangdong Provincial Key Laboratory of Quantum Science and Engineering, Southern University of Science and Technology, Shenzhen \\ 518055, China \\ ${ }^{3}$ Shenzhen Key Laboratory of Quantum Science and Engineering, Southern University of Science and Technology, Shenzhen 518055, China \\ ${ }^{4}$ Graduate School of Mathematics, Nagoya University, Chikusa-ku, Nagoya 464-8602, Japan
}

(Received 6 November 2019; accepted 30 June 2020; published 15 July 2020)

\begin{abstract}
Quantum communication over network is particularly appealing in a similar way to classical network communication, but a partially corrupted quantum network has not been sufficiently studied. We discuss quantum state transmission over a partially corrupted quantum network, in which the sender transmits $m_{0}$ qudits, i.e., $d$-level systems, to the receiver via network, but $m_{1}$ channels are corrupted. As our result, we show that the optimal transmission rate is at least $\left(m_{0}-2 m_{1}+1\right) \log d$ under the following two settings. In the first case, the unitaries on intermediate nodes are arbitrary and the corruptions on the $m_{1}$ channels are individual. In the second case, the unitaries on intermediate nodes are restricted to Clifford operations and the corruptions on the $m_{1}$ channels are adaptive, i.e., they are caused by an attack with a quantum memory. Further, our code in the second case realizes the noiseless communication even with the single-shot setting and is constructed dependently only on the network topology and the places of the $m_{1}$ corrupted channels.
\end{abstract}

DOI: 10.1103/PhysRevResearch.2.033079

\section{INTRODUCTION}

In the realm of quantum communication, a quantum network can be expected to work a basic infrastructure for quantum communication [1]. A quantum network is composed of edges, i.e., quantum communication channels to transmit quantum states, and nodes, i.e., quantum processors to convert input quantum states to output quantum state. Several coding schemes for a quantum network have been proposed for communication across many players [2-10]. A typical quantum network coding scheme was proposed in Ref. [3], and was already implemented experimentally as a photonic system [11]. Since a quantum network has several communication paths, it enhances robust communication between two players by the diversification of the risk when the quantum communication network is partially corrupted. Although the robust network communication against corruption has been discussed in the classical case by many papers [12-15], this type of research for quantum network are very limited, and only the preceding paper [16] showed the existence of such a network code on the restricted class of quantum networks. Unfortunately, their code construction is not practical due to their asymptotic construction, and the optimality of their transmission rate is not discussed.

\footnotetext{
*hayashi@ sustech.edu.cn

${ }^{\dagger}$ m17021a@math.nagoya-u.ac.jp

Published by the American Physical Society under the terms of the Creative Commons Attribution 4.0 International license. Further distribution of this work must maintain attribution to the author(s) and the published article's title, journal citation, and DOI.
}

This paper derives a simple expression for the optimal value of the reliable transmission rate, which is called the quantum capacity, on a more general class of quantum networks with corruption. In fact, no single-letterized formula is known for the quantum capacity of most of the channels because its existing formula requires a limiting expression [17-20]. Therefore, it is a quite difficult problem to derive the quantum capacity under a certain condition. Also, we propose a reliable quantum network code that realizes a perfect error correction under single transmission without the asymptotic construction when the quantum network satisfies a certain condition. In addition, our code achieves the optimal transmission rate, which is strictly higher than the achievable rate in the preceding paper [16]. While our code construction depends on the network structure and the place of the corruption, it does not depend on the corrupting operations. Whereas conventional network coding considers the optimization of node operations given a directed graph of the network, we consider the quantum capacity when node operations are given as well because it is often quite difficult to control node operations. Specifically, we address the worst-case capacity under a certain condition, which is formulated as follows.

Our model is described as follows. Every quantum channel transmits a $d$-dimensional system by one use of the network. The sender has $m_{0}$ outgoing quantum channels. Each intermediate node has the same number of incoming quantum channels and outgoing quantum channels. The node applies a fixed unitary across the incoming quantum systems and outputs them to the outgoing quantum channels. Finally, the receiver receives $m_{0}$ quantum systems via $m_{0}$ incoming quantum channels. The existing classical networks are composed of noiseless channel by applying error-correcting codes. Hence, existing studies on classical network coding address 
the network composed of noiseless channels unless they are corrupted [12-14]. Therefore, this paper also assumes that the channels are noiseless unless they are corrupted because the errors of these noncorrupted channels can be corrected by quantum error-correcting code. In particular, due to the no-cloning theorem of quantum system, if the dimension of output system is larger than the input system, we cannot avoid the corruption of quantum system. That is, such a node operation is not useful for a quantum network. Hence, each intermediate node is assumed to have the same dimensional output system as the input system. Also, the number of corrupted channels is assumed to be $m_{1}$, and the network is assumed to have no cycle and to be well synchronized, i.e., to have no delayed transmission. Only the sender and the receiver are allowed to optimize their coding operation due to the difficulty of node operation control. Although the paper [16] assumed that the sender and the receiver do not know the places of corrupted channels, the places are assumed to be known to them in our setting.

There are two types of quantum corruptions. The first one is the individual corruption, in which the corruption on each corrupted quantum channel is done individually. If the channel is simply broken due to an accident, the corruption belongs to this type. The other is the adaptive corruption, in which the corruptions on respective corrupted quantum channels are done adaptively. That is, the corruptions are caused by the attack with a quantum memory, and the quantum memory interacts with the corrupted quantum channel on each corruption. When the corruption is done by an adversarial attack, we need to consider the adaptive corruption. This kind of corruption can be written by quantum comb [21,22]. The adaptive corruption is more general than the individual corruption, and the adaptive corruption cannot be reduced to the individual corruption in general. For example, adaptive strategies cannot be reduced to individual strategies in quantum channel discrimination [23].

In this scenario, when all nodes carefully maintain their connected channels, they can find which channels are corrupted. It needs a larger cost to replace the corrupted installed channels by new noiseless channels because these corrupted channels are across physically distant nodes and the replacement of the channels takes longer times and requires many preparations. Also, the intermediate nodes might have physical space limitation so that the node operations are fixed. Hence, as a realistic solution, it is natural to employ the encoding and the decoding on the sender and receiver sides.

In the classical communication over the partially corrupted network, we can show that the capacity, i.e., the maximum transmission rate, is not smaller than $\left(m_{0}-\right.$ $\left.m_{1}\right) \log d$ [13]. In contrast, when our quantum channel has only individual corruptions, we find that the quantum capacity, i.e., the maximum transmission rate of the quantum state, is not smaller than $\left(m_{0}-2 m_{1}+1\right) \log d$. This fact is shown by the analysis of coherent information on the quantum network. Further, when the unitaries on intermediate nodes are limited to Clifford operations, the quantum capacity is not smaller than $\left(m_{0}-2 m_{1}+1\right) \log d$ even when the corruptions are adaptive while the achievable rate in the preceding paper [16] is $\left(m_{0}-2 m_{1}\right) \log d$.
In this case, our code can be constructed in the singleshot setting by using Clifford operations. This construction depends only on Clifford operations of intermediate nodes and the places of the corrupted channels, and is independent of Eve's operation to the corrupted channels. Our code construction to achieve the capacity can be intuitively explained in the case of Clifford operations as follows.

We can characterize the $m_{1}$ corruptions by the symplectic structure as follows. When we apply a suitable symplectic matrix, the first corruption can be written as the corruption on the first qudit, i.e., the corrupted computation base and the corrupted Fourier base are given as the first base. However, at the other corruptions, the corrupted computation bases and the corrupted Fourier bases split in general. Hence, in the worst case, totally $2 m_{1}-1$ quantum systems are corrupted. Since the symplectic diagonalization extracts noncorrupted bases, it enables us to propose our code construction to achieve the capacity.

The remaining parts of this paper are organized as follows. First, for a comparison, Sec. II discusses the classical network. Next, Sec. III addresses the general unitary network model with the first type of corruption. Then, with the second type of corruption, Sec. IV considers the network model whose node operation is limited to Clifford operations. As its special class, this section also discusses the basis-linear network model, in which the unitary operation is given as a linear operation with respect to the basis. Finally, Sec. V describes a concrete example of a network model.

\section{CLASSICAL NETWORK MODEL}

When all the node operations are invertible and linear on a finite field of order $q(=d)$, the receiver can find a linear subspace for corrupted information, as discussed in Ref. [13]. The dimension of the subspace is bounded by $m_{1}$. Hence, the capacity is not smaller than $\left(m_{0}-m_{1}\right) \log d$. However, when node operations are not necessarily linear but are invertible, we cannot apply the above discussion. Even in this case, we can show that the capacity is greater than or equal to $\left(m_{0}-\right.$ $\left.m_{1}\right) \log d$ as follows.

Assume that every channel transmits a system whose number of elements is $d$ by one use of the network and the sender has $m_{0}$ outgoing channels. Node operations are not necessarily linear but are invertible. $m_{1}$ channels are corrupted. Hence, we can assume that $m_{1}$ corruptions are done sequentially. Let $X_{i-1}^{\prime}$ and $X_{i}$ be the whole information before and after the $i$ th corruption, respectively. We denote the input and output information of this network by $X_{0}$ and $X_{m_{1}}^{\prime}$, respectively. Then, $X_{i}^{\prime}$ is written as $f_{i}\left(X_{i}\right)$ by using an invertible function $f_{i}$. Since the channel capacity of classical communication is given by the maximum mutual information between the input information and the output information, it is sufficient to show that

$$
I\left(X_{0} ; X_{m_{1}}^{\prime}\right)=I\left(X_{0} ; X_{m_{1}}\right) \geqslant\left(m_{0}-m_{1}\right) \log d
$$

with a certain distribution $P_{X_{0}}$ of $X_{0}$. 


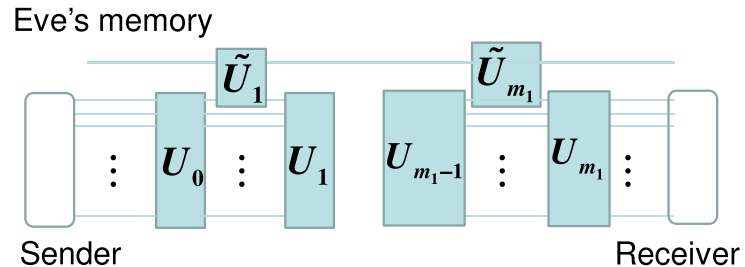

FIG. 1. Unitary network with adaptive corruption. After the application of the unitary $U_{i-1}$, the unitary $\tilde{U}_{i}$ is applied.

Now, we set the distribution $P_{X_{0}}$ of $X_{0}$ to be the uniform distribution, which implies that

$$
H\left(X_{0}\right)=m_{0} \log d .
$$

From the network structure, we find the relation $H\left(X_{i} \mid X_{i+1}\right) \leqslant$ $\log d$. The chain rule of conditional entropy implies that

$$
\begin{aligned}
H\left(X_{0} \mid X_{m_{1}}\right) & \leqslant H\left(X_{0} \ldots X_{m_{1}-1} \mid X_{m_{1}}\right) \\
& =\sum_{l=0}^{m_{1}-1} H\left(X_{l} \mid X_{l+1} X_{l+2} \ldots X_{m_{1}}\right) \\
& \leqslant \sum_{l=0}^{m_{1}-1} H\left(X_{l} \mid X_{l+1}\right) \leqslant m_{1} \log d .
\end{aligned}
$$

The combination of (2) and (3) yields (1).

\section{GENERAL UNITARY NETWORK MODEL}

The general unitary network model is described as follows. In this model, we assume that the places of the channels to be corrupted are known. Since our network is composed of unitary operations and partial corruptions, our network model of the adaptive corruption is given as the general form with Fig. 1, whose reason is illustrated in Fig. 2. The input and output systems are the $m_{0}$-tensor product system $\mathcal{H}^{\otimes m_{0}}$ of the same system $\mathcal{H}$ of dimension $d$, and $m_{1}+1$ unitaries $\boldsymbol{U}=$ $\left(U_{0}, U_{1}, \ldots, U_{m_{1}}\right)$ are applied between the input and output systems, which has $m_{1}$ intervals. Eve can access only the first system on each interval, and has her memory so that the corruption in the $i$ th interval is given as the unitary $\tilde{U}_{i}$ between her memory and the corrupted system, i.e., the first system on the $i$ th interval.

Our first result is on the minimum capacity of the general unitary network with individual corruption, in which Eve is assumed to have no memory. Hence, her operation on the $i$ th interval can be written as TP-CP maps $\Gamma=\left(\Gamma_{1}, \ldots, \Gamma_{m_{1}}\right)$ as Fig. 3. In this case, the channel between the input and output systems is denoted by $\Lambda(\boldsymbol{U}, \boldsymbol{\Gamma}) . C(\Lambda)$ expresses the quantum capacity of a quantum channel $\Lambda$. The following theorem characterizes the quantum capacity under the individual corruption.

Theorem 1. The minimum quantum capacity is given as follows:

$$
\min C(\Lambda(\boldsymbol{U}, \boldsymbol{\Gamma}))=\left(m_{0}-2 m_{1}+1\right) \log d .
$$

Here, the minimum is taken over all channels $\Lambda(\boldsymbol{U}, \boldsymbol{\Gamma})$ under the individual corruption.

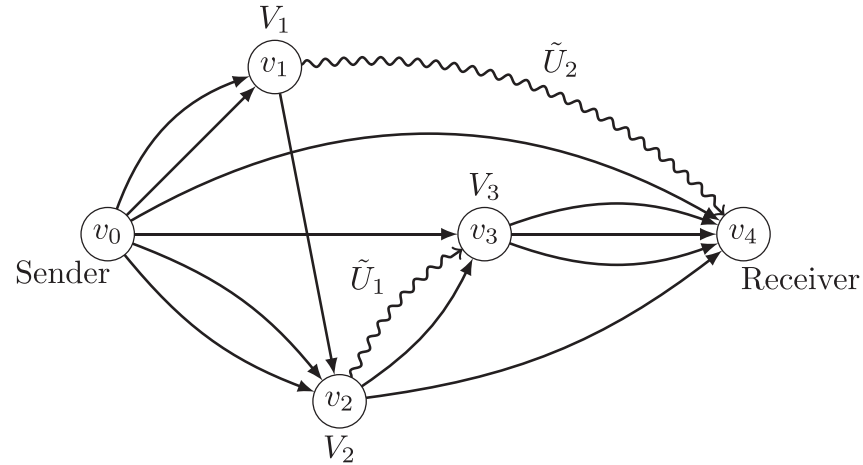

(a)

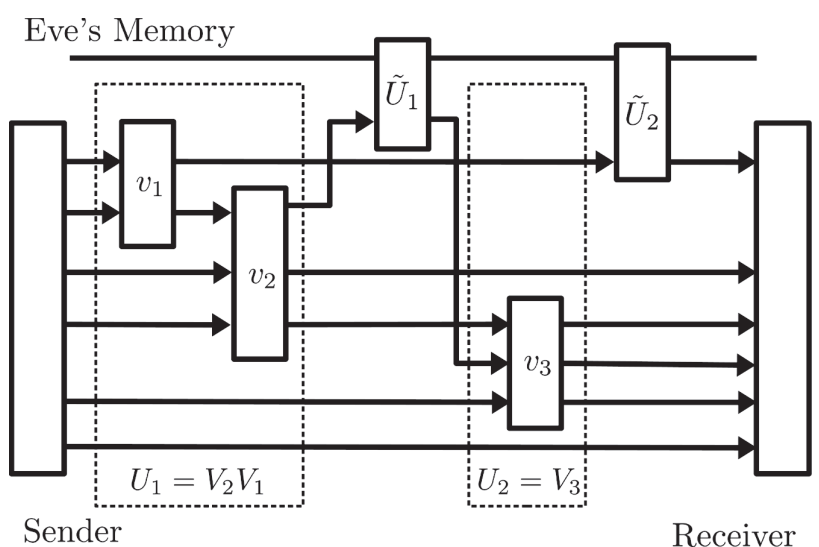

(b)

FIG. 2. Reorganization of the network in the form of Fig. 1. (a) Quantum network with three intermediate nodes. Sender and receiver have $m_{0}=6$ outgoing and incoming channels, respectively, and each intermediate node has the same number of incoming and outgoing channels. The zigzagged lines are corrupted channels and two channels are corrupted (i.e., $m_{1}=2$ ). (b) The network in (a) in the form of Fig. 1.

To show the theorem, we employ the coherent information $I_{c}(\rho, \Lambda)$ for an input state $\rho$ and a quantum channel $\Lambda$ with an output system $A$. By using the environment $E$ of $\Lambda$, the coherent information $I_{c}(\rho, \Lambda)$ is written as $H(A)-H(E)$, where $H(A)$ and $H(E)$ are the von Neumann entropy of the respective system when the input state is $\rho$ [24], [25, (8.37)]. It is known that the quantum capacity $C(\Lambda)$ is given as the maximum

$$
\lim _{n \rightarrow \infty} \max _{\rho} \frac{1}{n} I_{c}\left(\rho, \Lambda^{\otimes n}\right),
$$

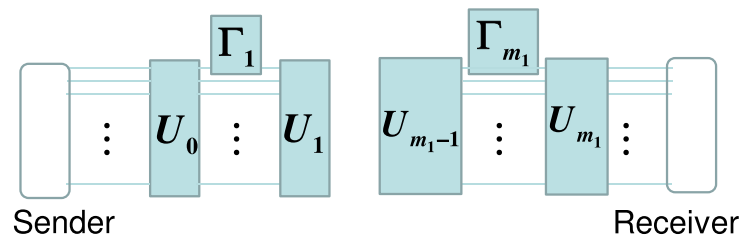

FIG. 3. Unitary network with individual corruption. After the application of the unitary $U_{i-1}$, the noisy operation $\Gamma_{i}$ is applied. 


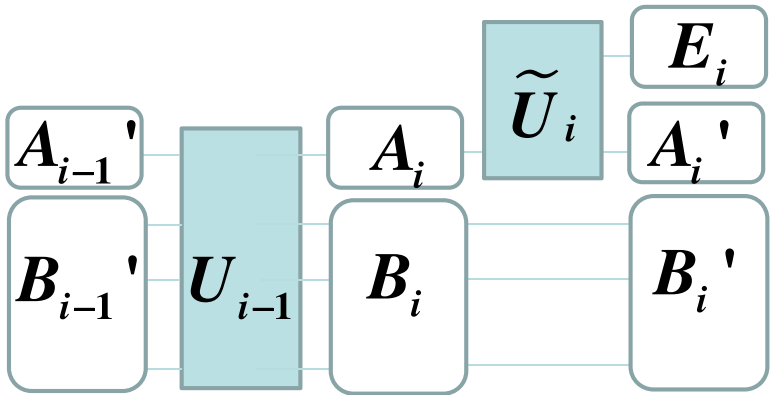

FIG. 4. Relation among systems $A_{i-1}^{\prime}, B_{i-1}^{\prime}, A_{i}, B_{i}, A_{i}^{\prime}, B_{i}^{\prime}$, and $E_{i}$. After the application of the unitary $U_{i-1}$, the operation $\tilde{U}_{i}$ is applied.

where the maximum is taken over all the input densities on the $n$-tensor system of the input system of $\Lambda$ [17-20], [25, Theorem 9.10].

Lemma 1. An individual corruption $\Lambda(\boldsymbol{U}, \boldsymbol{\Gamma})$ satisfies

$$
\max _{\rho} I_{c}(\rho, \Lambda(\boldsymbol{U}, \boldsymbol{\Gamma})) \geqslant\left(m_{0}-2 m_{1}+1\right) \log d .
$$

The inequality $\geqslant$ in Eq. (4) follows from Lemma 1, the additivity property $I_{c}\left(\rho^{\otimes n}, \Lambda^{\otimes n}\right)=n I_{c}(\rho, \Lambda)$, and the above capacity formula (5). In the remaining sections, we will show the existence of a channel $\Lambda(\boldsymbol{U}, \boldsymbol{\Gamma})$ to satisfy the equality of (4) in the Clifford network model, whose formulation will be given in the next section. Since a Clifford network is a special case of unitary networks, this existence completes the proof of Theorem 1.

Proof of Lemma 1. It is sufficient to show the case when $U_{0}$ is the identity matrix. Let $\rho_{\text {mix }, m_{0}-1}$ be the completely mixed state on $\mathcal{H}^{\otimes m_{0}-1}$. We set the initial state to be $|0\rangle\langle 0| \otimes$ $\rho_{\text {mix }, m_{0}-1}$.

Consider the time after the unitary $U_{i-1}$ is applied but $\Gamma_{i}$ is not applied yet. At this time, we denote the systems to be attacked and the remaining systems by $A_{i}$ and $B_{i}$, respectively. After the application of $\Gamma_{i}$, we denote the systems to be attacked and the remaining systems by $A_{i}^{\prime}$ and $B_{i}^{\prime}$, respectively. We consider Steinspring representation $\tilde{U}_{i}$ of $\Gamma_{i}$, in which the output of the environment is $E_{i}$. Figure 4 summarizes the relation among the systems $A_{i-1}^{\prime}, B_{i-1}^{\prime}, A_{i}, B_{i}, A_{i}^{\prime}, B_{i}^{\prime}$, and $E_{i}$.

Since the state on $A_{1}^{\prime} E_{1}$ is pure, we have $H\left(A_{1}^{\prime}\right)=H\left(E_{1}\right)$. Hence, we have

$$
\begin{aligned}
& H\left(A_{1}^{\prime} B_{1}^{\prime}\right)-H\left(E_{1}\right)=H\left(A_{1}^{\prime}\right)+H\left(B_{1}^{\prime}\right)-H\left(E_{1}\right) \\
& \quad=H\left(B_{1}^{\prime}\right)=\left(m_{0}-1\right) \log d .
\end{aligned}
$$

As shown later, for $i=2, \ldots, m_{1}$, we have

$$
H\left(A_{i}^{\prime} B_{i}^{\prime}\right)-H\left(E_{i}\right) \geqslant H\left(A_{i-1}^{\prime} B_{i-1}^{\prime}\right)-2 \log d .
$$

Combining (7) and (8), we have

$$
\begin{aligned}
& I_{c}\left(|0\rangle\langle 0| \otimes \rho_{\text {mix }, m_{0}-1}, \Lambda\left(U_{0}, \Gamma_{1}, U_{1}, \ldots, \Gamma_{m_{1}}, U_{m_{1}}\right)\right) \\
& \quad=H\left(A_{m_{1}}^{\prime} B_{m_{1}}^{\prime}\right)-H\left(E_{1}, \ldots, E_{m_{1}}\right) \\
& \quad \geqslant\left(m_{0}-2 m_{1}+1\right) \log d .
\end{aligned}
$$

Now, we show (8). Consider the purification of $\rho_{A_{i}^{\prime} E_{i}}$ by using the reference system $R$. Then, $H(R)=H\left(A_{i}^{\prime} E_{i}\right)=$ $H\left(A_{i}\right)$. Since $H\left(A_{i}\right)+H\left(A_{i}^{\prime}\right)-H\left(E_{i}\right)=H(R)+H\left(A_{i}^{\prime}\right)-$
$H\left(R A_{i}^{\prime}\right)=I\left(R ; A_{i}^{\prime}\right) \geqslant 0$, we have

$$
H\left(E_{i}\right) \leqslant H\left(A_{i}\right)+H\left(A_{i}^{\prime}\right) .
$$

Thus,

$$
\begin{aligned}
H\left(A_{i}^{\prime} B_{i}^{\prime}\right)-H\left(E_{i}\right) \\
\quad \stackrel{(a)}{\geqslant} H\left(A_{i}^{\prime} B_{i}^{\prime}\right)-H\left(A_{i}\right)-H\left(A_{i}^{\prime}\right) \\
=H\left(B_{i}^{\prime}\right)+H\left(A_{i}^{\prime}\right)-H\left(A_{i}\right)-H\left(A_{i}^{\prime}\right)-I\left(A_{i}^{\prime} ; B_{i}^{\prime}\right) \\
=H\left(A_{i}^{\prime} B_{i}^{\prime} E_{i}\right)-H\left(A_{i}^{\prime} E_{i}\right)+I\left(A_{i}^{\prime} E_{i} ; B_{i}^{\prime}\right) \\
\quad+H\left(A_{i}^{\prime}\right)-H\left(A_{i}\right)-H\left(A_{i}^{\prime}\right)-I\left(A_{i}^{\prime} ; B_{i}^{\prime}\right) \\
\quad \stackrel{(b)}{=} H\left(A_{i} B_{i}\right)-H\left(A_{i}\right)+I\left(E_{i} ; B_{i}^{\prime} \mid A_{i}^{\prime}\right)-H\left(A_{i}\right) \\
\quad \stackrel{(c)}{\geqslant} H\left(A_{i} B_{i}\right)-2 H\left(A_{i}\right)=H\left(A_{i-1}^{\prime} B_{i-1}^{\prime}\right)-2 H\left(A_{i}\right) \\
\geqslant
\end{aligned}
$$

Here, $(a)$ follows from (10), $(b)$ follows from $H\left(A_{i}^{\prime} B_{i}^{\prime} E_{i}\right)=$ $H\left(A_{i} B_{i}\right)$ and $H\left(A_{i}^{\prime} E_{i}\right)=H\left(A_{i}\right)$, and (c) follows from $I\left(E_{i} ; B_{i}^{\prime} \mid A_{i}^{\prime}\right) \geqslant 0$.

\section{CLIFFORD NETWORK MODEL}

\section{A. Fundamental limit of quantum capacity in Clifford network model}

As pointed out in Ref. [13], in the classical network, when node operations are composed of linear operations, the corruption can be corrected by linear operations in the sender and receiver sides. As a quantum version of a network composed of linear operations, we introduce Clifford network models. In this model, we can expect a simple construction of error correction. Indeed, we can construct a code to achieve the capacity even with the single-shot setting in this case. Also, Theorem 1 can be extended to adaptive corruptions described in Fig. 1. (A concrete example of an adaptive corruption is given in Sec. V.) For this aim, we prepare several notations. Given a prime power $q=p^{d_{q}}$, our Hilbert space $\mathcal{H}$ is assumed to be spanned by the computational basis $\{|x\rangle\}_{x \in \mathbb{F}_{q}}$, where $\mathbb{F}_{q}$ is the algebraic extension of the finite field $\mathbb{F}_{p}$ with degree $d_{q}$. That is, the dimension of the Hilbert space $\mathcal{H}$ is assumed to be $q$. Then, for $s, t \in \mathbb{F}_{q}$, we define the generalized Pauli operators $\mathrm{X}(s)$ and $\mathrm{Z}(t)$ as $\mathrm{X}(s):=\sum_{x \in \mathbb{F}_{q}}|x+s\rangle\langle x|$ and $\mathbf{Z}(t):=\sum_{x \in \mathbb{F}_{q}} \omega^{\operatorname{tr} x t}|x\rangle\langle x|$, where $\omega:=e^{2 \pi \sqrt{-1} / p}$. Here, for an element $z \in \mathbb{F}_{q}$, $\operatorname{tr} z$ expresses the element $\operatorname{Tr} M_{z} \in \mathbb{F}_{p}$, where $M_{z}$ denotes the matrix representation of the multiplication map $x \mapsto z x$ with identifying the finite field $\mathbb{F}_{q}$ with the vector space $\mathbb{F}_{p}^{d_{q}}$. We define the Fourier basis $\left\{|y\rangle_{F} \in \mathcal{H}\right\}_{y \in \mathbb{F}_{q}}$ of the computational basis $\{|x\rangle\}_{x \in \mathbb{F}_{q}} \in \mathcal{H}$ as

$$
|y\rangle_{F}:=\sum_{x \in \mathbb{F}_{q}} \frac{1}{\sqrt{q}} \omega^{\operatorname{tr} x y}|x\rangle .
$$

To consider our network model, for vectors $\mathbf{s}=$ $\left(s_{1}, \ldots, s_{n}\right), \mathbf{t}=\left(t_{1}, \ldots, t_{n}\right) \in \mathbb{F}_{q}^{n}$, we define the operators $\mathbf{X}(\mathbf{s})$ and $\mathbf{Z}(\mathbf{t})$ on the $n$-fold tensor product system $\mathcal{H}^{\otimes n}$ as $\mathbf{X}(\mathbf{s}):=\mathbf{X}\left(s_{1}\right) \otimes \cdots \otimes \mathbf{X}\left(s_{n}\right)$ and $\mathbf{Z}(\mathbf{t}):=\mathbf{Z}\left(t_{1}\right) \otimes$ $\cdots \otimes \mathbf{Z}\left(t_{n}\right)$. Then, the discrete Weyl operator is defined as $\mathbf{W}(\mathbf{s}, \mathbf{t}):=\mathbf{X}(\mathbf{s}) \mathbf{Z}(\mathbf{t})$. Then, for $(\mathbf{s}, \mathbf{t}),\left(\mathbf{s}^{\prime}, \mathbf{t}^{\prime}\right) \in \mathbb{F}_{q}^{2 n}$, we define the skew-symmetric matrix $J$ on $\mathbb{F}_{q}^{2 n}$ and the 
inner product $\left\langle(\mathbf{s}, \mathbf{t}),\left(\mathbf{s}^{\prime}, \mathbf{t}^{\prime}\right)\right\rangle \in \mathbb{F}_{p}$ as $J=\left(\begin{array}{cc}0 & -I \\ I & 0\end{array}\right)$ with $\left\langle(\mathbf{s}, \mathbf{t}),\left(\mathbf{s}^{\prime}, \mathbf{t}^{\prime}\right)\right\rangle:=\sum_{i=1} \operatorname{tr}\left(s_{i} s_{i}^{\prime}+t_{i} t_{i}^{\prime}\right)$. Then, the commutation relation

$$
\mathbf{W}(\mathbf{s}, \mathbf{t}) \mathbf{W}\left(\mathbf{s}^{\prime}, \mathbf{t}^{\prime}\right)=\omega^{\left\langle(\mathbf{s}, \mathbf{t}), J\left(\mathbf{s}^{\prime}, \mathbf{t}^{\prime}\right)\right\rangle} \mathbf{W}\left(\mathbf{s}^{\prime}, \mathbf{t}^{\prime}\right) \mathbf{W}(\mathbf{s}, \mathbf{t})
$$

holds, and a square matrix $g$ on $\mathbb{F}_{q}^{2 n}$ is called a symplectic matrix when $\left\langle(\mathbf{s}, \mathbf{t}), J\left(\mathbf{s}^{\prime}, \mathbf{t}^{\prime}\right)\right\rangle=\left\langle g(\mathbf{s}, \mathbf{t}), J g\left(\mathbf{s}^{\prime}, \mathbf{t}^{\prime}\right)\right\rangle$ for $(\mathbf{s}, \mathbf{t}),\left(\mathbf{s}^{\prime}, \mathbf{t}^{\prime}\right) \in \mathbb{F}_{q}^{2 n}$.

Next, we introduce Clifford group as a subset of the set $\mathcal{U}\left(\mathcal{H}^{\otimes n}\right)$ of unitaries on $\mathcal{H}^{\otimes n}$. Using the set $\mathcal{W}:=$ $\{c \mathbf{W}(\mathbf{s}, \mathbf{t})\}_{|c|=1,(\mathbf{s}, \mathbf{t}) \in \mathbb{F}_{q}^{2 n}}$, we define the Clifford group $\mathcal{C}$ as $\mathcal{C}:=$ $\left\{U \in \mathcal{U}\left(\mathcal{H}^{\otimes n}\right) \mid U \mathcal{W} U^{-1}=\mathcal{W}\right\}$. An element of $\mathcal{C}$ is called a Clifford unitary.

For any element $U \in \mathcal{C}$, there exists a symplectic matrix $g$ such that

$$
U \mathbf{W}(\mathbf{s}, \mathbf{t}) U^{-1}=c \mathbf{W}(g(\mathbf{s}, \mathbf{t}))
$$

for $(\mathbf{s}, \mathbf{t}) \in \mathbb{F}_{q}^{2 n}$ with a complex number $c$ satisfying $|c|=1$. Conversely, for any symplectic matrix $g$, there exists a unitary $U$ to satisfy (13). A typical construction of such a unitary $U$ is given in [25, Sec. 8.3]. This construction is called metaplectic representation and is denoted by $U(g)$ in this paper.

Now, the input and output systems are assumed to be $\mathcal{H}^{\otimes m_{0}}$, and the unitary $U_{i}$ is to be an element of Clifford group. Such a network is called Clifford network. We choose a symplectic matrix $g_{i}$ as $U\left(g_{i}\right)=U_{i}$. The minimum capacity of Clifford networks is derived as follows.

Theorem 2. For Clifford network, the minimum quantum capacity is $\left(m_{0}-2 m_{1}+1\right) \log q$ in the adaptive corruption, i.e., the case when the corruption is caused by the attack with a quantum memory.

Since a Clifford network model is a special case of channels mentioned in Theorem 1, Theorem 2 shows the existence of a channel $\Lambda(\boldsymbol{U}, \boldsymbol{\Gamma})$ to satisfy the equality of (4) in Theorem 1. Hence, Theorem 2 implies the remaining part of Theorem 1 .

\section{B. Parametric characterization of quantum capacity}

To show Theorem 2, we introduce additional parameters to characterize the quantum capacity. For this aim, we describe the behaviors of the errors in the terms of the symplectic structure. The errors are described by vectors in $\mathbb{F}_{q}^{2 m_{0}}$. For this aim, we introduce notations and parameters of the network as follows. Let $e_{i}$ be the vector in $\mathbb{F}_{q}^{2 m_{0}}$ that has only one nonzero element 1 in the $i$ th entry. Using $e_{1}$ and $e_{m_{0}+1}$, we define $2 m_{1}$ vectors $v_{1}, \ldots, v_{2 m_{1}} \in \mathbb{F}_{q}^{2 m_{0}}$ as $v_{i}:=g_{0}^{-1} \cdots g_{i-1}^{-1} e_{1}$ and $v_{m_{1}+i}:=g_{0}^{-1} \cdots g_{i-1}^{-1} e_{m_{0}+1}$ for $i=1, \ldots, m_{1}$. Since $e_{1}$ and $e_{m_{0}+1}$ describe the directions of errors in the respective interval, all the directions in the linear space $\mathcal{V}$ spanned by $v_{1}, \ldots, v_{2 m_{1}}$ are corrupted in this whole network.

In this paper, when a matrix $P$ satisfies $P^{2}=P$ and $\operatorname{Im} P=$ $\mathcal{V}$, it is called a projection onto $\mathcal{V}$. Then, we choose a projection $P_{\mathcal{V}}$ onto $\mathcal{V}$. Since $P_{\mathcal{V}}^{\top} J P_{\mathcal{V}}$ is also an antisymmetric matrix, the rank of $P_{\mathcal{V}}^{\top} J P_{\mathcal{V}}$ is an even number. The rank of the matrix $P_{\mathcal{V}}^{\top} J P_{\mathcal{V}}$ equals the rank of matrix $\left(\left\langle v_{i}, J v_{j}\right\rangle\right)_{i, j}$. Hence, the rank of $P_{\mathcal{V}}^{\top} J P_{\mathcal{V}}$ does not depend on the choice of the projection $P_{\mathcal{V}}$ onto $\mathcal{V}$ while the choice of the projection $P_{\mathcal{V}}$ onto $\mathcal{V}$ is not unique. With these observations, we define the integers $m_{*}$ and $m_{* *}$ as $m_{*}=\left(\operatorname{rank} P_{\mathcal{V}}^{\top} J P_{\mathcal{V}}\right) / 2$ and $m_{* *}=\operatorname{dim} \mathcal{V}-m_{*}$.
As shown by using fundamental knowledge of stabilizer codes [26-29][30, Sec. 5.3] in Appendix B, the quantum capacity $C$ is characterized as follows.

Lemma 2 . The capacity $C$ is lower bounded as $C \geqslant\left(m_{0}-\right.$ $\left.m_{* *}\right) \log q$ in the adaptive case, i.e., the case when the corruption is caused by the attack with a quantum memory.

Proof of Lemma 2. To consider this problem, we employ a stabilizer code [26-29] [30, Sec. 5.3] after applying the unitary $U_{0}^{-1} U_{1}^{-1} \ldots U_{m_{1}}^{-1}$ to the received system. Define $\mathcal{V}_{1}:=\operatorname{Ker} P_{\mathcal{V}}^{\top} J P_{\mathcal{V}} \cap \mathcal{V}=\mathcal{V} \cap \mathcal{V}^{\perp}$, where $\mathcal{V}^{\perp}:=$ $\left\{\mathbf{x} \in \mathbb{F}_{q}^{2 m_{0}} \mid\langle\mathbf{x}, J \mathbf{y}\rangle=0\right.$ for any $\left.\mathbf{y} \in \mathcal{V}\right\}$. Due to symplectic diagonalization, we can choose $m_{*}$-independent vectors $w_{1}, \ldots, w_{m_{*}} \in \mathcal{V} \subset \mathbb{F}_{q}^{2 m_{0}}$ and other $m_{*}$-independent vectors $w_{1}^{\prime}, \ldots, w_{m_{*}}^{\prime} \in \mathcal{V} \subset \mathbb{F}_{q}^{2 m_{0}}$ such that $\left\langle w_{i}^{\prime}, J w_{j}\right\rangle=\delta_{i, j}$, $\left\langle w_{i}, J w_{j}\right\rangle=\left\langle w_{i}^{\prime}, J w_{j}^{\prime}\right\rangle=0$ for $i, j=1, \ldots, m_{*}$. (For symplectic diagonalization, see Appendix A.) We define $\mathcal{V}_{2}$ and $\mathcal{V}_{3}$ as the spaces spanned by $w_{1}, \ldots, w_{m_{*}}$ and $w_{1}^{\prime}, \ldots, w_{m_{*}}^{\prime}$, respectively. Then, we define the subspace $\mathcal{N}:=\mathcal{V}_{1}+\mathcal{V}_{2}$ with dimension $m_{* *}$. We find that $\mathcal{N}$ is self-orthogonal, i.e., $\mathcal{N} \subset \mathcal{N}^{\perp}$, where $\mathcal{N}^{\perp}:=\left\{\mathbf{x} \in \mathbb{F}_{q}^{2 m_{0}} \mid\langle\mathbf{x}, J \mathbf{y}\rangle=0\right.$ for any $\mathbf{y} \in$ $\mathcal{N}\}$. We define the projection $\varpi: \mathbb{F}_{q}^{2 m_{0}} \rightarrow \mathbb{F}_{q}^{2 m_{0}} / \mathcal{N}^{\perp}$. Since $\mathcal{N}^{\perp} \cap \mathcal{V}_{3}=\{\mathbf{0}\}$, the map $\varpi$ is injective on $\mathcal{V}_{3}$. That is, there exists a map $\tau$ from $\mathbb{F}_{q}^{2 m_{0}} / \mathcal{N}^{\perp}$ to $\mathbb{F}_{q}^{2 m_{0}}$ such that $\mathcal{V}_{3} \subset \operatorname{Im} \tau$, i.e., $\tau(\varpi(v))=v$ for any $v \in \mathcal{V}_{3}$. When we apply the stabilizer code with the correcting set $\operatorname{Im} \tau$, the set of correctable errors is $\operatorname{Im} \tau+\mathcal{N}$, which includes $\mathcal{V}$. (The detail of this error-correcting code is given in Appendix B.) That is, any error in $\mathcal{V}$ can be corrected. In this code, the logarithm of the dimension of the transmitted space is $\operatorname{dim} \mathbb{F}_{q}^{m_{0}} / \mathcal{N}=m_{0}-$ $m_{* *}$. Therefore, even for any adaptive corruption, the above stabilizer code can decode the original state $\rho$ on $\mathcal{H}^{\otimes m_{0}-m_{* *}}$. This discussion shows Lemma 2. Our code construction depends only on $g_{0}, \ldots, g_{m_{1}}$. That is, it is independent of the remaining unitaries $\tilde{U}_{1}, \ldots, \tilde{U}_{m_{1}}$ of Eve's corruption.

The lower bound of Lemma 2 is achieved by the following lemma.

Lemma 3. When Eve changes the state on the corrupted edge to the completely mixed state, the capacity $C$ equals $\left(m_{0}-m_{* *}\right) \log q$.

Proof of Lemma 3. Now, we consider the case when Eve changes the state on the corrupted edge to the completely mixed state. It is equivalent to the case when all errors in $\mathcal{V}=\mathcal{N}+\mathcal{V}_{3}$ happen with equal probability. Then,

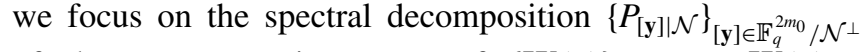
of the common eigenspace of $\{\mathbf{W}(\mathbf{x})\}_{\mathbf{x} \in \mathcal{N}}$ as $\mathbf{W}(\mathbf{x})=$ $\sum_{[\mathbf{y}] \in \mathbb{F}_{q}^{2 m_{0}} / \mathcal{N}^{\perp}} c_{[\mathbf{y}], \mathbf{x}} P_{[\mathbf{y}] \mid \mathcal{N}}$. The coherence between different eigenvalues in operators in $\mathcal{N}$ is collapsed. The rank of $P_{[\mathbf{y}] \mid \mathcal{N}}$ does not depend on $[\mathbf{y}] \in \mathbb{F}_{q}^{2 m_{0}} / \mathcal{N}^{\perp}$ and equals $q^{m_{0}-m_{* *}}$. Hence, the application of Lemma 8 to the subspace $\mathcal{N}$ given in the proof of Lemma 2 with $\mathcal{E}=\mathcal{V}_{3}$ shows Lemma 3. That is, it is impossible to transmit a space larger than $\operatorname{Im} P_{[0] \mid \mathcal{N}}$.

\section{Range of network parameters and basis-linear network model}

As described in Lemmas 2 and 3, the capacity is characterized by $\operatorname{rank} P_{\mathcal{V}}^{\top} J P_{\mathcal{V}}$ and $\operatorname{dim} \mathcal{V}$. The range of these network parameters can be summarized as the following lemma. 
Lemma 4. The following conditions are equivalent for two integers $l_{*}$ and $l_{* *}$

(1) $2 m_{1}-1 \geqslant l_{* *} \geqslant l_{*} \geqslant 1$ and $m_{0} \geqslant l_{* *}$.

(2) There exists a sequence of Clifford unitaries $U\left(g_{0}\right), \ldots, U\left(g_{m_{1}}\right) \quad$ such that $2 l_{*}=\operatorname{rank} P_{\mathcal{V}}^{\top} J P_{\mathcal{V}} \quad$ and $l_{*}+l_{* *}=\operatorname{dim} \mathcal{V}$.

Applying Lemma 4 to Lemmas 2 and 3 for the case of $m_{* *}=l_{* *}=2 m_{1}-1$, we obtain Theorem 2 . Lemma 4 will be shown in Sec. IV D. Although the relation $(2) \Rightarrow(1)$ of Lemma 4 can be directly shown, the proof of the opposite direction needs the detailed network construction stated in condition (2). For this aim, we introduce a special class of Clifford networks, called basis-linear networks.

In basis-linear networks, we assume that each Clifford unitary $U_{i}$ is characterized as the basis exchange caused by an invertible matrix $\bar{g}_{i}$ on $\mathbb{F}_{q}^{m_{0}}$, which is similar to the case of CSS (Calderbank-Shor-Steane) code [31,32]. That is, the Clifford unitary $U_{i}$ is given as the unitary $\bar{U}\left(\bar{g}_{i}\right)$ defined by $\bar{U}(\bar{g})|\mathbf{x}\rangle=|\bar{g} \mathbf{x}\rangle$. Its action on the Fourier basis $\left\{|\mathbf{y}\rangle_{F}\right\}_{\mathbf{y} \in \mathbb{F}_{q}^{m_{0}}}$ is characterized as $\bar{U}(\bar{g})|\mathbf{y}\rangle_{F}=\left|[\bar{g}]_{F} \mathbf{y}\right\rangle_{F}$, where $[\bar{g}]_{F}$ is defined as the transpose $\left(\bar{g}^{-1}\right)^{\top}$ of the inverse matrix $\bar{g}^{-1}[16$, Appendix A]. Hence, we have

$$
\bar{U}(\bar{g})=U\left[\left(\begin{array}{cc}
\bar{g} & 0 \\
0 & {[\bar{g}]_{F}}
\end{array}\right)\right] .
$$

Let $\bar{e}_{i}$ be the vector in $\mathbb{F}_{q}^{m_{0}}$ that has only one nonzero element 1 in the $i$ th entry. By using the vector $\bar{e}_{1}=$ $(1,0, \ldots, 0) \in \mathbb{F}_{q}^{m_{0}}$, the vectors $v_{1}, \ldots, v_{2 m_{1}}$ are written as $v_{i}=\left(\bar{v}_{i}, \mathbf{0}\right)$ and $v_{m_{1}+i}=\left(\mathbf{0}, \bar{v}_{i}^{\prime}\right)$ with $\bar{v}_{i}:=\bar{g}_{0}^{-1} \ldots \bar{g}_{i-1}^{-1} \bar{e}_{1}$ and $\bar{v}_{i}^{\prime}:=\bar{g}_{0}^{\top} \ldots \bar{g}_{i-1}^{\top} \bar{e}_{1}$ for $i=1, \ldots, m_{1}$. We define the matrices $\bar{V}$ and $\bar{V}^{\prime}$ as $\left(\bar{v}_{1}, \ldots, \bar{v}_{m_{1}}\right)$ and $\left(\bar{v}_{1}^{\prime}, \ldots, \bar{v}_{m_{1}}^{\prime}\right)$. Then, we have

$$
m_{*}=\operatorname{rank}\left(\bar{V}^{\prime}\right)^{\top} \bar{V}, \quad m_{* *}=\operatorname{rank} \bar{V}+\operatorname{rank} \bar{V}^{\prime}-m_{*} .
$$

Introducing new parameters $\left(l_{1}, l_{2}, l_{3}\right)$ satisfying $l_{*}=$ $l_{3}, l_{* *}=l_{1}+l_{2}-l_{3}$, we can characterize the possible range of these parameters under this submodel as follows.

Lemma 5. The following conditions are equivalent for three integers $l_{1}, l_{2}$, and $l_{3}$.

(a) $m_{1} \geqslant l_{1} \geqslant l_{3} \geqslant 1, m_{1} \geqslant l_{2} \geqslant l_{3} \geqslant 1$, and $m_{0} \geqslant l_{1}+$ $l_{2}-l_{3}$.

(b) There exists a sequence of invertible matrices $\bar{g}_{0}, \ldots, \bar{g}_{m_{1}}$ over finite field $\mathbb{F}_{q}$ such that $\operatorname{rank} \bar{V}=l_{1}$, $\operatorname{rank} \bar{V}^{\prime}=l_{2}$, and $\operatorname{rank}\left(\bar{V}^{\prime}\right)^{\top} \bar{V}=l_{3}$.

Lemma 5 will be shown in Sec. IV D as well as Lemma 4. Our proof of $(1) \Rightarrow(2)$ of Lemma 4 will be given by using (a) $\Rightarrow$ (b) of Lemma 5 .

\section{Proofs of Lemmas 4 and 5}

Our proofs of Lemmas 4 and 5 are composed of the parameter constraint part and the network construction part. The parameter constraint part proves the relations $(2) \Rightarrow(1)$ of Lemma 4 and (b) $\Rightarrow$ (a) of Lemma 5. The network construction part proves $(\mathrm{a}) \Rightarrow(\mathrm{b})$ of Lemma 5. Lastly, we show the relation $(1) \Rightarrow(2)$ of Lemma 4 using Lemma 5.

\section{Parameter constraint part}

The relations $(2) \Rightarrow(1)$ of Lemma 4 and (b) $\Rightarrow$ (a) of Lemma 5 show the constraint for the range of parameters $l_{*}$ and $l_{* *}$ to characterize our network. Before starting our proof of these relations, we prepare a more general discussion. Remind that $m_{*}$ and $m_{* *}$ are defined as $m_{*}=\left(\operatorname{rank} P_{\mathcal{V}}^{\top} J P_{\mathcal{V}}\right) / 2$ and $m_{* *}=\operatorname{dim} \mathcal{V}-m_{*}$. Since the rank of the submatrix $\left(\left\langle v_{i}, J v_{j}\right\rangle\right)_{i, j=1, m_{0}+1}$ is 2 , the rank of $\left(\left\langle v_{i}, J v_{j}\right\rangle\right)_{i, j=1}^{2 m_{1}}$ is at least 2. As the rank of $\left(\left\langle v_{i}, J v_{j}\right\rangle\right)_{i, j=1}^{2 m_{1}}$ equals the rank of $P_{\mathcal{V}}^{\top} J P_{\mathcal{V}}$, the inequality

$$
m_{*} \geqslant 1
$$

holds. Thus, the inequality $\operatorname{dim} \mathcal{V} \geqslant \operatorname{rank} P_{\mathcal{V}}^{\top} J P_{\mathcal{V}}$ implies

$$
m_{* *} \geqslant m_{*} \text {. }
$$

So, since $2 m_{1} \geqslant m_{* *}+m_{*}$, we have

$$
m_{* *} \leqslant 2 m_{1}-1 \text {. }
$$

Now we prove $(2) \Rightarrow(1)$ of Lemma 4 . The inequality $l_{* *} \geqslant l_{*}$ follows from (17), and the inequality $l_{*} \geqslant 1$ follows from (16). Since rank $P_{\mathcal{V}}^{\top} J P_{\mathcal{V}}=2 l_{*}$, there exist $l_{* *}$ independent vectors $x_{1}, \ldots, x_{l_{* *}} \in \mathcal{V}$ such that $\left\langle x_{j}, J x_{i}\right\rangle=0$ for $i, j=$ $1, \ldots l_{* *}$. Since the number of such vectors is upper bounded by $m_{0}$, we have $m_{0} \geqslant l_{* *}$. The relation $2 m_{1}-1 \geqslant l_{* *}$ follows from (18). Hence, we have the relation $(2) \Rightarrow(1)$ of Lemma 4.

Next, we show (b) $\Rightarrow$ (a) of Lemma 5. Since the relations $m_{1} \geqslant l_{1} \geqslant l_{3}, m_{1} \geqslant l_{2} \geqslant l_{3}$ are trivial, it is sufficient to show $m_{0} \geqslant l_{1}+l_{2}-l_{3}$ and $l_{3} \geqslant 1$. Since the matrix $\left(\bar{V}^{\prime}\right)^{\top} \bar{V}$ is not zero, we have $l_{3} \geqslant 1$. Hence, the relations (15) and (18) imply the relation $m_{0} \geqslant l_{1}+l_{2}-l_{3}$ as

$$
\begin{aligned}
m_{0} \geqslant 2 m_{1}-1 \geqslant m_{* *} & =\operatorname{rank} \bar{V}+\operatorname{rank} \bar{V}-m_{*} \\
& =\operatorname{rank} \bar{V}+\operatorname{rank} \bar{V}-\operatorname{rank}\left(\bar{V}^{\prime}\right)^{\top} \bar{V} \\
& =l_{1}+l_{2}-l_{3} .
\end{aligned}
$$

Thus, we obtain the relation $(b) \Rightarrow(a)$ of Lemma 5 .

\section{Network construction part}

Now, we show (a) $\Rightarrow$ (b) of Lemma 5 . That is, we concretely construct our network based on the parameters $l_{1}, l_{2}$, and $l_{3}$. We assume that $l_{1} \geqslant l_{2}$. Otherwise, we can exchange the computation basis and the Fourier basis. We choose $\bar{g}_{m_{1}}$ to be the identity matrix. For $i=1, \ldots, m_{1}$, we choose $\bar{g}_{i-1}$ to be $A_{i}^{-1} A_{i-1}$, where the matrix $A_{i}$ is defined as follows. The constructed matrices $\bar{V}, \bar{V}^{\prime},\left(\bar{V}^{\prime}\right)^{\top} \bar{V}$ are described in Fig. 5.

$A_{0}$ is the identity matrix. For $i=1, \ldots, l_{3}$, we define $A_{i}$ as the transposition between the first entry and the $i$ th entry. For $i=l_{1}+1, \ldots, m_{1}$, we define $A_{i}$ as the identity matrix.

For $i=1, \ldots, l_{2}-l_{3}$, we define $A_{l_{3}+i}$ in the following way. For the first, $\left(l_{3}+2 i-1\right)$ th, and $\left(l_{3}+2 i\right)$ th entries, it is defined as $\left(\begin{array}{lll}1 & 0 & 1 \\ 1 & 1 & 0 \\ 0 & 0 & 1\end{array}\right)$. For other indices $j, j^{\prime}$, the matrix component $\left(A_{l_{3}+i}\right)_{j, j^{\prime}}$ is defined as $\delta_{j, j^{\prime}}$.

For $i=1, \ldots, l_{1}-l_{2}$, we define $A_{l_{2}+i}$ in the following way. For the first and $\left(2 l_{2}-l_{3}+i\right)$ th entries, it is defined as $\left(\begin{array}{ll}1 & 0 \\ 1 & 1\end{array}\right)$. For other indices $j, j^{\prime}$, the matrix component $\left(A_{l_{2}+i}\right)_{j, j^{\prime}}$ is defined as $\delta_{j, j^{\prime}}$.

Then, for $i=1, \ldots, l_{3}$, we have $\bar{v}_{i}=\bar{e}_{i}$. For $i=1, \ldots, l_{2}-l_{3}$, we have $\bar{v}_{l_{3}+i}=\bar{e}_{1}+\bar{e}_{l_{3}+2 i-1}$. For $i=1, \ldots, l_{1}-l_{2}$, we have $\bar{v}_{l_{2}+i}=\bar{e}_{1}+\bar{e}_{2 l_{2}-l_{3}+i}$. For $i=l_{1}+1, \ldots, m_{1}$, we have $\bar{v}_{i}=\bar{e}_{1}$.

The matrix $\left[A_{l_{3}+i}\right]_{F}$ is characterized for $i=1, \ldots, l_{2}-$ $l_{3}$ as follows. For the first, $\left(l_{3}+2 i-1\right)$ th, and $\left(l_{3}+2 i\right)$ th 

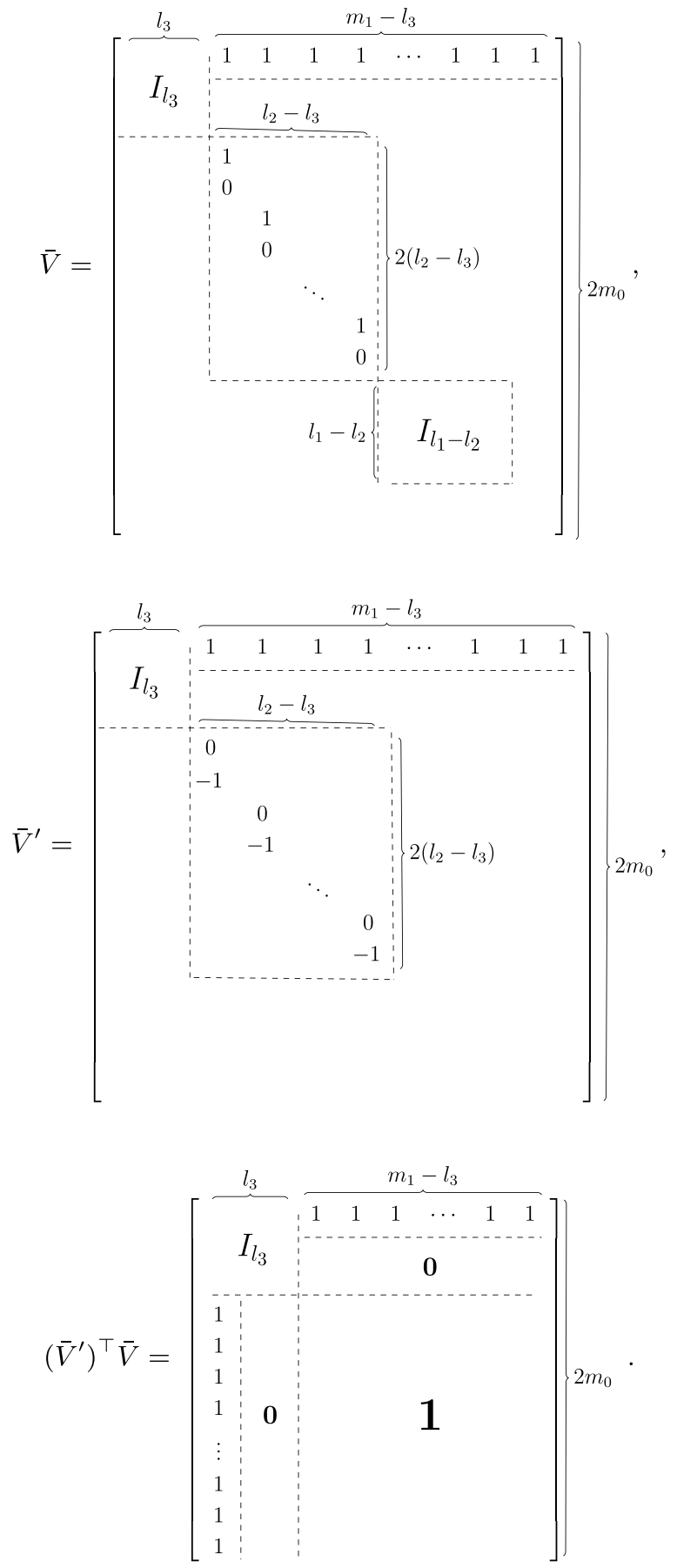

FIG. 5. Matrices $\bar{V}, \bar{V}^{\prime},\left(\bar{V}^{\prime}\right)^{\top} \bar{V}$ of network construction. 0 (1) is the matrix with all 0 (1) elements, and the elements of empty cells are 0 .

entries, it is given as $\left(\begin{array}{ccc}1 & -1 & 0 \\ 0 & 1 & 0 \\ -1 & 0 & 1\end{array}\right)$. For other indices $j, j^{\prime}$, $\left(\left[A_{l_{3}+i}\right]_{F}\right)_{j, j^{\prime}}$ is given as $\delta_{j, j^{\prime}}$.

The matrix $\left[A_{l_{2}+i}\right]_{F}$ is characterized for $i=1, \ldots, l_{1}-l_{2}$ as follows. For the first and $\left(2 l_{2}-l_{3}+i\right)$ th entries, it is given as $\left(\begin{array}{cc}1 & -1 \\ 0 & 1\end{array}\right)$. For other indices $j, j^{\prime}$, the matrix component $\left(\left[A_{l_{2}+i}\right]_{F}\right)_{j, j^{\prime}}$ is given as $\delta_{j, j^{\prime}}$. Then, for $i=1, \ldots, l_{3}$, we have $\bar{v}_{i}^{\prime}=\bar{e}_{i}$. For $i=1, \ldots, l_{2}-l_{3}$, we have $\bar{v}_{l_{3}+i}^{\prime}=\bar{e}_{1}-$ $\bar{e}_{l_{3}+2 i}$. For $i=1, \ldots, l_{1}-l_{2}$, we have $\bar{v}_{l_{2}+i}^{\prime}=\bar{e}_{1}$. For $i=l_{1}+$

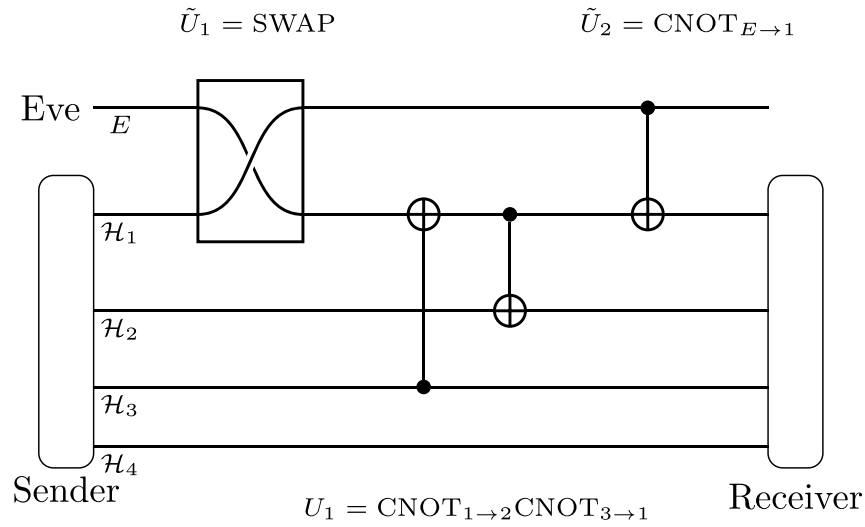

FIG. 6. Adaptive attack for the worst case with $m_{1}=2$ and $m_{0}=4$.

$1, \ldots, m_{1}$, we have $\bar{v}_{i}^{\prime}=\bar{e}_{1}$. Therefore, we have $\operatorname{rank} \bar{V}=l_{1}$ and $\operatorname{rank} \bar{V}^{\prime}=l_{2}$.

Also, when $j=2, \ldots, l_{3}$ or $j^{\prime}=2, \ldots, l_{3}$, we have $\left(\left(\bar{V}^{\prime}\right)^{\top} \bar{V}\right)_{j, j^{\prime}}=\delta_{j, j^{\prime}}$. When $j=1, l_{3}+1, \ldots, m_{1} \quad$ and $j^{\prime}=1, l_{3}+1, \ldots, m_{1}$, we have $\left(\left(\bar{V}^{\prime}\right)^{\top} \bar{V}\right)_{j, j^{\prime}}=1$. Hence, $\operatorname{rank}\left(\bar{V}^{\prime}\right)^{\top} \bar{V}=l_{3}$. Thus, we obtain the relation $(\mathrm{a}) \Rightarrow(\mathrm{b})$ of Lemma 5.

\section{Proof of $(1) \Rightarrow(2)$ of Lemma 4}

We choose $\left(l_{1}, l_{2}, l_{3}\right):=\left(1, l_{*}+l_{* *}-1, l_{*}\right)$. Then, $\left(l_{1}, l_{2}, l_{3}\right)$ satisfies condition (a) of Lemma 4 . We also have $(a) \Rightarrow(b)$ from Sec. IV D 2. From (15), the condition (b) of Lemma 4 implies the existence of Clifford network with $\left(m_{*}, m_{* *}\right):=\left(l_{3}, l_{1}+l_{2}-l_{3}\right)=\left(l_{*}, l_{* *}\right)$, which implies $(1) \Rightarrow(2)$ of Lemma 5 . Therefore, our proof of Lemmas 4 and 5 is completed.

\section{CONCRETE EXAMPLE FOR ADAPTIVE CORRUPTION}

To clarify the usefulness of our result, we discuss a concrete example for the worst capacity with $m_{0}=4$ and $m_{1}=2$ in the framework of Fig. 1. Also, we show a concrete example for adaptive corruption in this case. Consider the case given in Lemma 5 with $d=2, m_{0}=4$, and $m_{1}=2$. That is, we focus on the case given in Sec. IV D 2 with $l_{1}=l_{2}=2$ and $l_{3}=1$. This discussion is also helpful to understand the construction given in Sec. IV D 2.

As shown in Fig. 6, we set $U_{0}=U_{2}=I$ and $U_{1}=$ $\mathrm{CNOT}_{1 \rightarrow 2} \mathrm{CNOT}_{3 \rightarrow 1}$. In this case, the capacity is 1 , and only the fourth qubit is noiseless. Hence, the use of the fourth qubit is the best error correction to achieve capacity.

As a typical example of Eve's corruption, this figure illustrates the following adaptive corruption on a network. Eve corrupts each channel between the sender and the intermediate node and between the intermediate node and the receiver. Eve has one qubit system as her memory $E$. Eve applies the swap gate on $\mathcal{H}_{1} \otimes E$ at the first corruption and the CNOT gate on $E \otimes \mathcal{H}_{1}$, where $\mathcal{H}_{1}$ is the first qubit system. 
Finally to see that this case realizes the worst capacity, we show the relations $l_{1}=l_{2}=2$ and $l_{3}=1$. We notice that

$$
A_{0}=A_{1}=I, \quad A_{2}=\left(\begin{array}{lll}
1 & 0 & 1 \\
1 & 1 & 0 \\
0 & 0 & 1
\end{array}\right),
$$

$\bar{g}_{0}=\bar{g}_{2}=I$, and the first three entries of $\bar{g}_{1}=A_{1} A_{2}^{-1}$ are

$$
\left(\begin{array}{lll}
1 & 0 & 1 \\
1 & 1 & 1 \\
0 & 0 & 1
\end{array}\right)=\left(\begin{array}{lll}
1 & 0 & 0 \\
1 & 1 & 0 \\
0 & 0 & 1
\end{array}\right)\left(\begin{array}{lll}
1 & 0 & 1 \\
0 & 1 & 0 \\
0 & 0 & 1
\end{array}\right) .
$$

Since $\bar{g}_{1}$ and $\bar{g}_{1}^{-1}$ are

$$
\bar{g}_{1}=\left(\begin{array}{llll}
1 & 0 & 1 & 0 \\
1 & 1 & 1 & 0 \\
0 & 0 & 1 & 0 \\
0 & 0 & 0 & 1
\end{array}\right), \quad \bar{g}_{1}^{-1}=\left(\begin{array}{llll}
1 & 0 & 1 & 0 \\
1 & 1 & 0 & 0 \\
0 & 0 & 1 & 0 \\
0 & 0 & 0 & 1
\end{array}\right),
$$

we have

$$
\bar{V}=\left(\begin{array}{ll}
1 & 1 \\
0 & 1 \\
0 & 0 \\
0 & 0
\end{array}\right), \quad \bar{V}^{\prime}=\left(\begin{array}{ll}
1 & 1 \\
0 & 0 \\
0 & 1 \\
0 & 0
\end{array}\right), \quad\left(\bar{V}^{\prime}\right)^{\top} \bar{V}=\left(\begin{array}{ll}
1 & 1 \\
1 & 1
\end{array}\right),
$$

which implies $m_{* *}=\operatorname{rank} \bar{V}+\operatorname{rank} \bar{V}^{\prime}-\operatorname{rank}\left(\bar{V}^{\prime}\right)^{\top} \bar{V}=3$. Since $m_{0}-2 m_{1}+1=m_{0}-m_{* *}=1$, the capacity of this network is the minimum capacity. The optimal code for this case is to transmit the state only on the fourth channel of the network regardless of Eve's operations $\tilde{U}_{1}, \tilde{U}_{2}$.

\section{DISCUSSION}

We have shown that the quantum capacity is not smaller than $\left(m_{0}-2 m_{1}+1\right) \log d$ when the sender has $m_{0}$ outgoing channels, the receiver has $m_{0}$ incoming channels, each intermediate node applies invertible unitary, only $m_{1}$ channels are corrupted in our quantum network model, and other noncorrupted channels are noiseless. Our result holds with the following two cases. In the first case, the unitaries on intermediate nodes are arbitrary and the corruptions on the $m_{1}$ channels are individual. In the second case, the unitaries on intermediate nodes are restricted to Clifford operations and the corruptions on the $m_{1}$ channels are adaptive, i.e., they are caused by the attack allowed to have a quantum memory. Further, our code in the second case realizes the noiseless communication even with the single-shot setting, and depends only on the node operations, the network topology, and the places of the $m_{1}$ corrupted channels. That is, it is independent of Eve's operation on the $m_{1}$ corrupted channels. This code utilizes the following structure of this model. The error in the first corrupted channel can be concentrated to one quantum system. However, the errors of the computation basis and the Fourier basis in another corrupted channel split into two quantum systems in general. Hence, $2 m_{1}-1$ quantum systems are corrupted in the worst case. The first case has been shown by the analysis of the coherent information, and symplectic structure including symplectic diagonalization on the discrete system plays a key role in the second case. It is an interesting remaining problem to derive the quantum capacity when the operations on intermediate nodes are arbitrary unitaries and the corruptions on the $m_{1}$ channels are adaptive.

\section{ACKNOWLEDGMENTS}

M.H. is grateful to Professor M. Owari and Dr. G. Kato for helpful comments. M.H. was supported in part by Guangdong Provincial Key Laboratory (Grant No. 2019B121203002), Japan Society for the Promotion of Science (JSPS) Grant-inAid for Scientific Research (A) Grants No. 17H01280, (B) No. 16KT0017, and Kayamori Foundation of Informational Science Advancement. S.S. is supported by Lotte Foundation and JSPS Grant-in-Aid for JSPS Fellows No. JP20J11484.

\section{APPENDIX A: SYMPLECTIC DIAGONALIZATION}

For the choice of vectors $w_{1}, \ldots, w_{m_{*}}$ and $w_{1}^{\prime}, \ldots, w_{m_{*}}^{\prime}$, we summarize fundamental knowledge for symplectic diagonalization in the finite-dimensional system. Assume that $\mathcal{V}$ is a finite-dimensional vector space over a finite field $\mathbb{F}_{q}$. We consider a bilinear form $Q$ from $\mathcal{V} \times \mathcal{V}$ to $\mathbb{F}_{q}$. In the following discussion, $Q$ is not necessarily nondegenerate. Given an element $v \in \mathcal{V}, Q(v, \cdot)$ can be regarded as an element of the dual space $\mathcal{V}^{*}$ of $\mathcal{V}$. In this sense, $Q$ can be regarded as a linear map from $\mathcal{V}$ to $\mathcal{V}^{*}$. A bilinear form $Q$ from $\mathcal{V} \times \mathcal{V}$ to $\mathbb{F}_{q}$ is called antisymmetric when $Q\left(v_{1}, v_{2}\right)=-Q\left(v_{2}, v_{1}\right)$ and $Q\left(v_{1}, v_{1}\right)=0$ for $v_{1}, v_{2} \in \mathcal{V}$.

Lemma 6. Consider an antisymmetric bilinear form $Q$. Then, the rank of $Q$ is an even number $2 k$. There exists a basis $w_{1}, \ldots, w_{k}, w_{1}^{\prime}, \ldots, w_{k}^{\prime} \in \mathcal{V}$ such that $Q\left(w_{i}^{\prime}, w_{j}\right)=\delta_{i, j}$ and $Q\left(w_{i}, w_{j}\right)=Q\left(w_{i}^{\prime}, w_{j}^{\prime}\right)=0$ for $i, j=1, \ldots, k$.

This statement can be generalized as follows.

Lemma 7. Consider an antisymmetric bilinear form $Q$. Then, the rank of $Q$ is an even number $2 k$. Assume that $l+l^{\prime}$ linearly independent vectors $w_{1}, \ldots, w_{l}, w_{1}^{\prime}, \ldots, w_{l^{\prime}}^{\prime} \in$ $\mathcal{V}$ with $l \leqslant l^{\prime}<k$ satisfy $Q\left(w_{i}^{\prime}, w_{j^{\prime}}^{\prime}\right)=\delta_{i, j^{\prime}}$ and $Q\left(w_{i}, w_{j}\right)=$ $Q\left(w_{i^{\prime}}^{\prime}, w_{j^{\prime}}^{\prime}\right)=0$ for $i, j=1, \ldots, l$ and $i^{\prime}, j^{\prime}=1, \ldots, l^{\prime}$. There exist $2 k-l-l^{\prime}$ vectors $w_{l+1}, \ldots, w_{k}, w_{l^{\prime}+1}^{\prime}, \ldots, w_{k}^{\prime} \in$ $\mathcal{V}$ such that $Q\left(w_{i}^{\prime}, w_{j}\right)=\delta_{i, j}$ and $Q\left(w_{i}, w_{j}\right)=Q\left(w_{i}^{\prime}, w_{j}^{\prime}\right)=0$ for $i, j=1, \ldots, k$.

Proof of Lemma 7. First, we show that there exists a vector $w_{l+1} \in \mathcal{V}$ such that $Q\left(w_{i}^{\prime}, w_{j^{\prime}}\right)=\delta_{i, j^{\prime}}$ and $Q\left(w_{i}, w_{j}\right)=$ $Q\left(w_{i^{\prime}}^{\prime}, w_{j^{\prime}}^{\prime}\right)=0$ for $i, j=1, \ldots, l+1$ and $i^{\prime}, j^{\prime}=1, \ldots, l^{\prime}$ and $w_{l+1}$ is linearly independent of $w_{1}, \ldots, w_{l}, w_{1}^{\prime}, \ldots, w_{l^{\prime}}^{\prime}$.

When $l^{\prime}=l$, it is sufficient to choose one vector $w_{l+1}$ to satisfy $Q\left(w_{l+1}, w_{i}\right)=Q\left(w_{l+1}, w_{i}^{\prime}\right)=0$ for $i=1, \ldots, l$. Since the number of constraints is $2 l$ and is smaller than the dimension of the dual space of $\mathcal{V}$, we can choose such a vector. Since any linear combination of $w_{1}, \ldots, w_{l}, w_{1}^{\prime}, \ldots, w_{l^{\prime}}^{\prime}$ does not satisfy this condition, $w_{l+1}$ is linearly independent of $w_{1}, \ldots, w_{l}, w_{1}^{\prime}, \ldots, w_{l^{\prime}}^{\prime}$.

When $l^{\prime}>l$, it is sufficient to choose one vector $w_{l+1}$ to satisfy $Q\left(w_{l+1}, w_{i}\right)=Q\left(w_{l+1}, w_{i^{\prime}}^{\prime}\right)=0$ for $i=1, \ldots, l$ and $i^{\prime}=1, \ldots, l, l+2, \ldots, l^{\prime}$ and $Q\left(w_{l+1}, w_{l+1}^{\prime}\right)=1$. Since the number of constraints is $l+l^{\prime}$ and is smaller than the dimension, we can choose such a vector. Since any linear combination of $w_{1}, \ldots, w_{l}, w_{1}^{\prime}, \ldots, w_{l^{\prime}}^{\prime}$ does not satisfy this condition, $w_{l+1}$ is linearly independent of $w_{1}, \ldots, w_{l}, w_{1}^{\prime}, \ldots, w_{l^{\prime}}^{\prime}$.

When $l^{\prime}=l$, we exchange vectors $w_{1}, \ldots, w_{l+1}$ and $w_{1}^{\prime}, \ldots, w_{l}^{\prime}$ and repeat the above procedure. Otherwise, we repeat the above procedure. Therefore, we can choose the desired vectors inductively. 


\section{APPENDIX B: STABILIZER CODE}

\section{Construction of stabilizer code}

First, we summarize the fundamental knowledge for the stabilizer code on the system $\mathcal{H}^{\otimes m_{0}}$. Here, we assume that the Weyl operator $\mathbf{W}(\mathbf{x})$ is defined in the same way as Sec. IV. Although it was formulated in Refs. [26-29], the following discussion is based on the notation in [30, Sec. 5.3]. When a subspace $\mathcal{N} \subset \mathbb{F}_{q}^{2 m_{0}}$ satisfies the condition $\mathcal{N} \subset \mathcal{N}^{\perp}:=\{\mathbf{x} \in$ $\mathbb{F}_{q}^{2 m_{0}} \mid\langle\mathbf{x}, J \mathbf{y}\rangle=0$ for any $\left.\mathbf{y} \in \mathcal{N}\right\}$, it is called self-orthogonal. We consider the spectral decomposition $\left\{P_{[\mathbf{y}] \mid \mathcal{N}}\right\}_{[\mathbf{y}] \in \mathbb{F}_{q}^{2 m_{0}} / \mathcal{N}^{\perp}}$ for the common eigenspaces of $\{\mathbf{W}(\mathbf{x})\}_{\mathbf{x} \in \mathcal{N}}$ as $\mathbf{W}(\mathbf{x})=$ $\sum_{[\mathbf{y}] \in \mathbb{F}_{q}^{2 m_{0}} / \mathcal{N}^{\perp}} c_{[\mathbf{y}], \mathbf{x}} P_{[\mathbf{y}] \mid \mathcal{N}}$. Here, $c_{[\mathbf{y}], \mathbf{x}}$ is the eigenvalue and $P_{[\mathbf{y}] \mid \mathcal{N}}$ is the projection to the common eigenspace.

Given a self-orthogonal subspace $\mathcal{N} \subset \mathbb{F}_{q}^{2 m_{0}}$, we choose a map $\tau$ from $\mathbb{F}_{q}^{2 m_{0}} / \mathcal{N}^{\perp}$ to $\mathbb{F}_{q}^{2 m_{0}}$ such that $\tau([\mathbf{y}]) \in[\mathbf{y}]$ for $[\mathbf{y}] \in$ $\mathbb{F}_{q}^{2 m_{0}} / \mathcal{N}^{\perp}$. Then, the stabilizer code with the correcting set $\operatorname{Im} \tau$ is given as follows. The sender sets the initial state in the subspace $\operatorname{Im} P_{[0] \mid \mathcal{N}}$. After receiving the system, the receiver applies the projective measurement $\left\{P_{[\mathbf{y}] \mid \mathcal{N}}\right\}_{[\mathbf{y}] \in \mathbb{F}_{q}^{2 m_{0}} / \mathcal{N}^{\perp}}$. Then, when we observe $[\mathbf{y}] \in \mathbb{F}_{q}^{2 m_{0}} / \mathcal{N}^{\perp}$, the resultant state belongs to $\operatorname{Im} P_{[\mathbf{y}] \mid \mathcal{N}}$. We apply the unitary $\mathbf{W}(-\tau([\mathbf{y}]))$ so that the state is transferred from $\operatorname{Im} P_{[\mathbf{y}] \mid \mathcal{N}}$ to $\operatorname{Im} P_{[0] \mid \mathcal{N}}$. When the error is $\mathbf{W}(\tau([\mathbf{y}]))$, it can be corrected in this error correction. Also, when the error belongs to $\{\mathbf{W}(\mathbf{x}) \mid \mathbf{x} \in \mathcal{N}\}$, it does not change the subspace $\operatorname{Im} P_{[0] \mid \mathcal{N}}$. Hence, when the error belongs to $\left\{\mathbf{W}(\tau([\mathbf{y}])+\mathbf{x}) \mid[\mathbf{y}] \in \mathbb{F}_{q}^{2 m_{0}} / \mathcal{N}^{\perp}, \mathbf{x} \in \mathcal{N}\right\}$, it can be corrected.

\section{Capacity}

We consider another space $\mathcal{E} \subset \mathbb{F}_{q}^{2 m_{0}}$ such that $\mathcal{E} \cap \mathcal{N}^{\perp}=$ $\{\boldsymbol{0}\}$ and $\mathcal{E} \subset \mathcal{E}^{\perp}$. We define the projection $\varpi: \mathbb{F}_{q}^{2 m_{0}} \rightarrow$ $\mathbb{F}_{q}^{2 m_{0}} / \mathcal{N}^{\perp}$. The map $\varpi$ is injective on $\mathcal{E}$. Hence, we choose $\tau$ to satisfy $\mathcal{E} \subset \operatorname{Im} \tau$.

Now, we consider the case when all errors in $\mathcal{N}+\mathcal{E}$ happen with equal probability. To address this case, we focus on the noisy channel $\rho \mapsto \Lambda(\rho):=$ $\sum_{\mathbf{x} \in \mathcal{N}+\mathcal{E}} \frac{1}{|\mathcal{N}+\mathcal{E}|} \mathbf{W}(\mathbf{x}) \rho \mathbf{W}(\mathbf{x})^{\dagger}$. In this case, an error $\mathbf{x}$ in $\mathcal{E}$ moves the state from $P_{[\mathbf{0}] \mid \mathcal{N}}$ to $P_{[\mathbf{x}] \mid \mathcal{N}}$. Then, as shown later, we have

$$
\Lambda(\rho)=\frac{1}{|\mathcal{E}|} \sum_{\substack{\mathbf{x} \in \mathcal{E},[\mathbf{y}] \in \mathbb{F}_{q}^{2 m_{0}} / \mathcal{N}^{\perp}}} \mathbf{W}(\mathbf{x}) P_{[\mathbf{y}] \mid \mathcal{N}} \rho P_{[\mathbf{y}] \mid \mathcal{N}} \mathbf{W}(\mathbf{x})^{\dagger} .
$$

To derive (B1), we prepare several notations. Let $k$ and $l$ be the dimensions of $\mathcal{E}$ and $\mathcal{N}$, respectively. From the definition, we have $k \leqslant l$. Let $w_{1}^{\prime}, \ldots, w_{k}^{\prime}$ be a basis of $\mathcal{E}$, which automatically satisfies the condition that $\left\langle w_{i^{\prime}}^{\prime}, J w_{j^{\prime}}^{\prime}\right\rangle=0$ for $i^{\prime}, j^{\prime}=$ $1, \ldots, k$. Since the $k$ nonzero linear maps $\mathbf{x} \mapsto\left\langle\mathbf{x}, J w_{1}^{\prime}\right\rangle, \ldots$, $\mathbf{x} \mapsto\left\langle\mathbf{x}, J w_{k}^{\prime}\right\rangle$ on $\mathcal{N}$ are linearly independent, considering the dual basis of the above linear maps, we can choose $l$ vectors $w_{1}, \ldots, w_{l}$ of $\mathcal{N}$ such that $\left\langle w_{i}, J w_{j^{\prime}}^{\prime}\right\rangle=\delta_{i, j^{\prime}},\left\langle w_{i}, J w_{j}\right\rangle=0$ for $i, j=1, \ldots, l$ and $j^{\prime}=1, \ldots, k$. According to Lemma 7 , we choose vectors $w_{l+1}, \ldots, w_{m_{0}}, w_{k+1}^{\prime}, \ldots, w_{m_{0}}^{\prime} \in \mathbb{F}_{q}^{2 m_{0}}$. Then, we choose a symplectic matrix $g$ such that $g e_{i}=$ $w_{i}^{\prime}$ and $g e_{i+m_{0}}=w_{i}$ for $i=1, \ldots, m_{0}$, where $e_{i}$ is defined in Sec. IV. Hence, $U(g)\{c \mathbf{W}(\mathbf{x})|\mathbf{x} \in \mathcal{N}| c \mid,=1\} U(g)^{-1}$ equals $\left\{c \mathbf{W}_{A}(\mathbf{0}, \mathbf{t})\left|\mathbf{t} \in \mathbb{F}_{q}^{l},\right| c \mid=1\right\}$, where $\mathcal{H}_{A}:=\mathcal{H}^{\otimes l}$ and $\mathbf{W}_{A}(\mathbf{x})$ is the discrete Weyl operator on $\mathcal{H}_{A}$. With a $k$ dimensional subspace $\tilde{\mathcal{E}} \subset \mathbb{F}_{q}^{l}, U(g)\{c \mathbf{W}(\mathbf{x})|\mathbf{x} \in \mathcal{E}| c \mid,=$ $1\} U(g)^{-1}$ equals $\left\{c \mathbf{W}_{A}(\mathbf{s}, \mathbf{0})|\mathbf{s} \in \tilde{\mathcal{E}}| c \mid,=1\right\}$. We define the system $\mathcal{H}_{B}:=\mathcal{H}^{\otimes m_{0}-l}$ so that $\mathcal{H}^{\otimes m_{0}}=\mathcal{H}_{A} \otimes \mathcal{H}_{B}$, and denote the discrete Weyl operator on $\mathcal{H}_{B}$ by $\mathbf{W}_{B}(\mathbf{x})$. In this notation, $\operatorname{Im} P_{[\mathbf{x}] \mid \mathcal{N}}$ is the same dimensional system as $\mathcal{H}_{B}$.

Now, instead of the original system $\mathcal{H}^{\otimes m_{0}}$, we focus on the system $\mathcal{H}_{A} \otimes \mathcal{H}_{B}$ by applying the unitary $U(g)$. The noisy channel $\Lambda$ is unitarily equivalent to $\Lambda_{A} \otimes \iota_{B}$, where $\iota_{B}$ is the noiseless channel on $\mathcal{H}_{B}$ and $\Lambda_{A}$ is given as

$$
\begin{gathered}
\sum_{\mathbf{s} \in \tilde{\mathcal{E}}} \sum_{\mathbf{t} \in \mathbb{F}_{q}^{l}} \frac{1}{q^{l+k}} \mathbf{W}_{A}(\mathbf{s}, \mathbf{0}) \mathbf{W}_{A}(\mathbf{0}, \mathbf{t}) \rho \mathbf{W}_{A}(\mathbf{0}, \mathbf{t})^{\dagger} \mathbf{W}_{A}(\mathbf{s}, \mathbf{0})^{\dagger} \\
=\sum_{\mathbf{s} \in \tilde{\mathcal{E}}} \frac{1}{q^{k}} \sum_{\mathbf{t} \in \mathbb{F}_{q}^{l}} \mathbf{W}_{A}(\mathbf{s}, \mathbf{0})|\mathbf{t}\rangle\langle\mathbf{t}|\rho| \mathbf{t}\rangle\langle\mathbf{t}| \mathbf{W}_{A}(\mathbf{s}, \mathbf{0})^{\dagger} .
\end{gathered}
$$

That is, the relation $U(g)^{\dagger} \Lambda\left(U(g) \rho U(g)^{\dagger}\right) U(g)=\left(\Lambda_{A} \otimes\right.$ $\left.\iota_{B}\right)(\rho)$ holds for any state $\rho$. The symbol $[\mathbf{y}] \in \mathbb{F}_{q}^{2 m_{0}} / \mathcal{N}^{\perp}$ in Eq. (B1) corresponds to $\mathbf{t} \in \mathbb{F}_{q}^{l}$ in Eq. (B2), and $P_{[\mathbf{y}] \mid \mathcal{N}}$ in Eq. (B1) corresponds to $|\mathbf{t}\rangle\langle\mathbf{t}| \otimes I_{B}$. Hence, $U(g)\left(\Lambda_{A} \otimes\right.$ $\left.\iota_{B}\right)\left(U(g)^{\dagger} \rho U(g)\right) U(g)^{\dagger}$ equals the right-hand side of (B1), which shows (B1).

Since the channel $\Lambda_{A}$ in $\mathcal{H}_{A}$ is entanglement breaking, it is impossible to protect a space larger than $\mathcal{H}_{B}$, i.e., $\operatorname{Im} P_{[0] \mid \mathcal{N}}$. Since the above stabilizer code protects the space $\operatorname{Im} P_{[0] \mid \mathcal{N}}$, the maximum dimension of the correctable space is $q^{m_{0}-\operatorname{dim} \mathcal{N}}$.

Now, we consider the case of $n$ times use of this channel, i.e., $\Lambda^{\otimes n}$, which is unitarily equivalent to $\left(\Lambda_{A} \otimes \iota_{B}\right)^{\otimes n}=$ $\Lambda_{A}^{\otimes n} \otimes \iota_{B}^{\otimes n}$. Since $\Lambda_{A}$ is an entanglement-breaking channel, using Lemma 9 given below, we have

$$
\begin{gathered}
\max _{\rho} I_{c}\left(\rho, \Lambda^{\otimes n}\right)=\max _{\rho} I_{c}\left(\rho, \Lambda_{A}^{\otimes n} \otimes \iota_{B}^{\otimes n}\right)=\max _{\tau} I_{c}\left(\tau, \iota_{B}^{\otimes n}\right) \\
=n \max _{\tau} I_{c}\left(\tau, \iota_{B}\right)=n\left(m_{0}-\operatorname{dim} \mathcal{N}\right) \log q,
\end{gathered}
$$

where $I_{c}(\rho, \Lambda)$ is the coherent information. Hence, we obtain the following lemma.

Lemma 8. When all errors in $\mathcal{N}+\mathcal{E}$ happen with equal probability, the capacity $C$ equals $\left(m_{0}-\operatorname{dim} \mathcal{N}\right) \log q$.

\section{Lemma for calculation of capacity}

Lemma 9. When a channel $\Lambda_{A}$ is entanglement breaking, a channel $\Lambda_{B}$ satisfies the condition

$$
\max _{\rho} I_{c}\left(\rho, \Lambda_{A} \otimes \Lambda_{B}\right)=\max _{\tau} I_{c}\left(\tau, \Lambda_{B}\right) .
$$

Proof of Lemma 9. Let $A$ and $B\left(A^{\prime}\right.$ and $\left.B^{\prime}\right)$ be the input (output) systems of $\Lambda_{A}$ and $\Lambda_{B}$, respectively. We choose a state $\rho_{A B}$ on $A B$. Let $C$ be the reference system of the state $\rho_{A B}$ so that $\rho_{A B C}$ is the purification of $\rho_{A B}$. Let $\rho^{\prime}$ be the output system on the whole system of $A^{\prime}, B^{\prime}$, and $C$.

Since $\Lambda_{A}$ is entanglement breaking, it is written as $\Lambda_{A}(\sigma)=\sum_{a} \rho_{A^{\prime}=a} \operatorname{Tr} M_{a} \sigma$, where $\left\{M_{a}\right\}$ is a POVM and $\operatorname{rank} M_{a}=1$. Hence, $\Lambda_{A}\left(\rho_{A B C}\right)$ is written as $\sum_{a} P_{A^{\prime}=a} \rho_{B C \mid A^{\prime}=a} \otimes \rho_{A^{\prime}=a}$, where $\quad P_{A^{\prime}=a}:=\operatorname{Tr} M_{a} \rho_{A} \quad$ and $\rho_{B C \mid A^{\prime}=a}:=\operatorname{Tr}_{A} M_{a} \rho_{A B C} / P_{A^{\prime}=a}$. Hence, rank $\rho_{B C \mid A^{\prime}=a}=1$.

Then, we denote $\Lambda_{B}\left(\rho_{B C \mid A^{\prime}=a}\right)$ by $\rho_{B^{\prime} C \mid A^{\prime}=a}^{\prime}$. The coherent information $I_{c}\left(\rho_{A B}, \Lambda_{A} \otimes \Lambda_{B}\right)$ equals $D\left(\rho_{A^{\prime} B^{\prime} C}^{\prime} \| \rho_{A^{\prime} B^{\prime}}^{\prime} \otimes I_{C}\right)$, 
which is evaluated as

$$
\begin{aligned}
D & \left(\rho_{A^{\prime} B^{\prime} C}^{\prime} \| \rho_{A^{\prime} B^{\prime}}^{\prime} \otimes I_{C}\right) \\
= & D\left(\sum_{a} P_{A^{\prime}=a} \rho_{B^{\prime} C \mid A^{\prime}=a}^{\prime} \otimes \rho_{A^{\prime}=a}\right. \\
& \left.\| \sum_{a} P_{A^{\prime}=a} \rho_{B^{\prime} \mid A^{\prime}=a}^{\prime} \otimes \rho_{A^{\prime}=a} \otimes I_{C}\right) \\
\leqslant & D\left(\sum_{a} P_{A^{\prime}=a} \rho_{B^{\prime} C \mid A^{\prime}=a}^{\prime} \otimes|a\rangle\langle a|\right. \\
& \left.\| \sum_{a} P_{A^{\prime}=a} \rho_{B^{\prime} \mid A^{\prime}=a}^{\prime} \otimes|a\rangle\langle a| \otimes I_{C}\right) \\
= & \sum_{a} P_{A^{\prime}=a} D\left(\rho_{B^{\prime} C \mid A^{\prime}=a}^{\prime} \| \rho_{B^{\prime} \mid A^{\prime}=a}^{\prime} \otimes I_{C}\right) .
\end{aligned}
$$

The inequality follows from the information processing inequality for the map $|a\rangle\langle a| \mapsto \rho_{A^{\prime}=a}$.
Since rank $\rho_{B C \mid A^{\prime}=a}=1$, the state $\rho_{B C \mid A^{\prime}=a}$ is a purification of $\rho_{B \mid A^{\prime}=a}$. Thus, $D\left(\rho_{B^{\prime} C \mid A^{\prime}=a}^{\prime} \| \rho_{B^{\prime} \mid A^{\prime}=a}^{\prime} \otimes I_{C}\right)$ equals the coherent information $I_{c}\left(\rho_{B \mid A^{\prime}=a}, \Lambda_{B}\right)$. Hence, we have

$$
I_{c}\left(\rho_{A B}, \Lambda_{A} \otimes \Lambda_{B}\right) \leqslant \sum_{a} P_{A^{\prime}=a} I_{c}\left(\rho_{B \mid A^{\prime}=a}, \Lambda_{B}\right),
$$

which implies that

$$
\max _{\rho} I_{c}\left(\rho, \Lambda_{A} \otimes \Lambda_{B}\right) \leqslant \max _{\tau} I_{c}\left(\tau, \Lambda_{B}\right) .
$$

Next, we show the converse inequality

$$
\max _{\rho} I_{c}\left(\rho, \Lambda_{A} \otimes \Lambda_{B}\right) \geqslant \max _{\tau} I_{c}\left(\tau, \Lambda_{B}\right) .
$$

For any state $\tau$ on the system $B$, define $\rho_{A B}=\rho_{A} \otimes \tau$ where $\rho_{A}$ is a pure state. Then, we have

$$
I_{c}\left(\rho_{A B}, \Lambda_{A} \otimes \Lambda_{B}\right)=I_{c}\left(\tau, \Lambda_{B}\right) .
$$

Therefore, we obtain (B7).
[1] H. J. Kimble, The quantum internet, Nature (London) 453, 1023 (2008).

[2] M. Hayashi, K. Iwama, H. Nishimura, R. Raymond, and S. Yamashita, Quantum network coding, in STACS 2007 SE-52, edited by W. Thomas and P. Weil, Vol. 4393 of Lecture Notes in Computer Science (Springer, Berlin, 2007), pp. 610-621.

[3] M. Hayashi, Prior entanglement between senders enables perfect quantum network coding with modification, Phys. Rev. A 76, 040301(R) (2007).

[4] H. Kobayashi, F. Le Gall, H. Nishimura, and M. Rötteler, General scheme for perfect quantum network coding with free classical communication, in Automata, Languages and Programming SE-52, edited by S. Albers, A. MarchettiSpaccamela, Y. Matias, S. Nikoletseas, and W. Thomas, Vol. 5555 of Lecture Notes in Computer Science (Springer, Berlin, 2009), pp. 622-633.

[5] D. Leung, J. Oppenheim, and A. Winter, Quantum network communication; The butterfly and beyond, IEEE Trans. Inf. Theory 56, 3478 (2010).

[6] H. Kobayashi, F. Le Gall, H. Nishimura, and M. Rotteler, Perfect quantum network communication protocol based on classical network coding, in Proceedings of 2010 IEEE International Symposium on Information Theory (ISIT) (IEEE, Piscataway, NJ, 2010), pp. 2686-2690.

[7] H. Kobayashi, F. Le Gall, H. Nishimura, and M. Rotteler, Constructing quantum network coding schemes from classical nonlinear protocols, in Proceedings of 2011 IEEE International Symposium on Information Theory (ISIT) (IEEE, Piscataway, NJ, 2011), pp. 109-113.

[8] A. Jain, M. Franceschetti, and D. A. Meyer, On quantum network coding, J. Math. Phys. 52, 032201 (2011).

[9] M. Owari, G. Kato, and M. Hayashi, Secure quantum network coding on butterfly network, Quantum Sci. Technol. 3, 014001 (2017).

[10] G. Kato, M. Owari, and M. Hayashi, Single-shot secure quantum network coding for general multiple unicast network with free public communication, in 10th International Conference on Information Theoretic Security (ICITS2017), edited by J. Shikata, Lecture Notes in Computer Science, Vol. 10681 (Springer, Berlin, 2017), pp. 166-187.

[11] H. Lu, Z.-D. Li, X.-F. Yin, R. Zhang, X.-X. Fang, L. Li, N.-L. Liu, F. Xu, Y.-A. Chen, and J.-W. Pan, Experimental quantum network coding, npj Quantum Inf. 5, 89 (2019).

[12] N. Cai and R. W. Yeung, Network error correction, Part 2: Lower bounds, Commun. Inf. Syst. 6, 37 (2006).

[13] S. Jaggi, M. Langberg, S. Katti, T. Ho, D. Katabi, M. Medard, and M. Effros, Resilient network coding in the presence of byzantine adversaries, IEEE Trans. Inf. Theory 54, 2596 (2008).

[14] H. Yao, D. Silva, S. Jaggi, and M. Langberg, Network codes resilient to jamming and eavesdropping, IEEE/ACM Trans. Networking 22, 1978 (2014).

[15] S. Song and M. Hayashi, Quantum network code for multipleunicast network with quantum invertible linear operations, in 13th Conference on the Theory of Quantum Computation, Communication and Cryptography (TQC 2018), edited by S. Jeffery, Leibniz International Proceedings in Informatics (LIPIcs), Vol 111 (University of Technology Sydney, Sydney, 2018), pp. 10:1-10:20.

[16] S. Song and M. Hayashi, Secure quantum network code without classical communication, IEEE Trans. Inf. Theory 66, 1178 (2020).

[17] S. Lloyd, The capacity of the noisy quantum channel, Phys. Rev. A, 55, 1613 (1997).

[18] H. Barnum, M. A. Nielsen, and B. Schumacher, Information transmission through a noisy quantum channel, Phys. Rev. A 57, 4153 (1998).

[19] P. W. Shor, The quantum channel capacity and coherent information, in Lecture Notes, MSRI Workshop on Quantum Computation, 2002, http://www.msri.org/publications/ln/msri/ 2002/quantumcrypto/shor/1/.

[20] I. Devetak, The private classical capacity and quantum capacity of a quantum channel, IEEE Trans. Inf. Theory 51, 44 (2005). 
[21] G. Chiribella, G. M. D'Ariano, and P. Perinotti, Quantum Circuit Architecture, Phys. Rev. Lett. 101, 060401 (2008).

[22] G. Chiribella, G. M. D'Ariano, and P. Perinotti, Theoretical framework for quantum networks, Phys. Rev. A 80, 022339 (2009).

[23] A. W. Harrow, A. Hassidim, D. W. Leung, and J. Watrous, Adaptive versus nonadaptive strategies for quantum channel discrimination, Phys. Rev. A 81, 032339 (2010).

[24] B. Schumacher and M. A. Nielsen, Quantum data processing and error correction, Phys. Rev. A 54, 2629 (1996).

[25] M. Hayashi, Quantum Information Theory, Graduate Texts in Physics (Springer, Berlin, 2017).

[26] A. R. Calderbank, E. M. Rains, P. W. Shor, and N. J. A. Sloane, Quantum Error Correction and Orthogonal Geometry, Phys. Rev. Lett. 78, 405 (1997).
[27] A. R. Calderbank, E. M. Rains, P. W. Shor, and N. J. A. Sloane, Quantum error correction via codes over GF(4), IEEE Trans. Inf. Theory 44, 1369 (1998).

[28] D. Gottesman, Class of quantum error-correcting codes saturating the quantum Hamming bound, Phys. Rev. A 54, 1862 (1996).

[29] M. Hamada, Notes on the fidelity of symplectic quantum errorcorrecting codes, Int. J. Quantum Inf. 01, 443 (2003).

[30] M. Hayashi, A Group Theoretic Approach to Quantum Information (Springer, Berlin, 2017).

[31] A. R. Calderbank and P. W. Shor, Good quantum error-correcting codes exist, Phys. Rev. A 54, 1098 (1996).

[32] A. M. Steane, Error Correcting Codes in Quantum Theory, Phys. Rev. Lett. 77, 793 (1996). 